\title{
USO DE ETIQUETAS DISCURSIVAS EN LA REDACCIÓN DE TEXTOS
}

\author{
USO DE ETIQUETAS DISCURSIVAS NA ESCRITA DE TEXTOS
}

USE OF SPEECH LABELS IN THE WRITING OF TEXTS

\author{
Marlenis MARTÍNEZ \\ Katihuska MOTA ${ }^{2}$ \\ Jennifer QUIÑONEZ ${ }^{3}$
}

RESUMEN: El propósito del estudio estuvo enmarcado en analizar las acciones que favorezcan el uso de etiquetas discursivas en la redacción de textos de los estudiantes del Colegio Particular San Javier, Comuna San Javier, Región del Maule-Chile. Metodológicamente se utilizó el enfoque cuantitativo, tipo campo con diseño descriptivo. Como hallazgos se diagnosticó el conocimiento que tienen los docentes y estudiantes sobre el uso de etiquetas discursivas para la redacción de textos; se identificaron las funciones más frecuentes que desempeñan las etiquetas discursivas en la redacción de textos escritos, se determinaron las deficiencias discursivas que produce el uso escaso o inapropiado de las mismas; finalmente, se definieron las acciones que favorecen la incorporación de las etiquetas discursivas en la redacción de textos por los educandos objeto de estudio.

PALABRAS CLAVE: Etiquetas. Discursiva. Redacción. Textos.

RESUMO: O objetivo do estudo se enquadrou na análise das ações que favorecem o uso de rótulos discursivos na redação de textos dos alunos da Escola Particular San Javier, Comuna de San Javier, Região do Maule-Chile. Metodologicamente, utilizou-se a abordagem quantitativa, tipo de campo com desenho descritivo. Como constatações, diagnosticou-se o conhecimento que professores e alunos possuem sobre o uso de rótulos discursivos para a escrita de textos; Foram identificadas as funções mais frequentes desempenhadas pelos rótulos discursivos na escrita de textos escritos, determinadas as deficiências discursivas produzidas por seu uso escasso ou inadequado; Por fim, foram definidas as ações que favorecem a incorporação de rótulos discursivos na escrita dos textos pelos alunos em estudo.

1 Universidad Miguel de Cervantes (UMC), Chile. Académico Dirección de Postgrado e Investigación. Postdoctorado em Gestión del conocimiento (Universidad Pedagógica Experimental Libertador). Doctorado en Ciencias de la Educación (Universidad Fermín Toro). Magíster en Educación, Mención Gerencia Educacional (Universidad Pedagógica Experimental Libertador-Instituto Pedagógico de Barquisimeto). Licenciada en Educación Integral, Mención Castellano y Literatura y Ciencias Naturales (Universidad Experimental de los Llanos Occidentales "Ezequiel Zamora” (UNELLEZ). ORCID. https://orcid.org/0000-0002-5926-599X. E-mail: marlenism3@gmail.com

${ }^{2}$ Universidad Miguel de Cervantes (UMC), Chile. Académica, Dirección de Postgrado e Investigación. Doctora en Ciencias de la Educación, Magister en Gerencia de Empresas mención Operaciones, Ingeniero de Petróleo. ORCID: https://orcid.org/0000-0002-4108-957X. E-mail: motakt@gmail.com

${ }^{3}$ Universidad Miguel de Cervantes (UMC), Chile. Académica, Dirección de Postgrado e Investigación. Magister en Ciencias de la Educación Superior mención Docencia Universitaria, Licenciada en educación Integral, Abogada. ORCID: https://orcid.org/0000-0003-3724-0182.E-mail: jennifer.zurina@hotmail.com

RPGE- Revista on line de Política e Gestão Educacional, Araraquara, v. 25, n. 1, p. 240-253, jan./abr. $2021 . \quad$ e-ISSN:1519-9029 DOI: https://doi.org/10.22633/rpge.v25i1.14727 
PALAVRAS-CHAVE: Rótulos. Discursivo. Escrita. Textos.

ABSTRACT: The purpose of the research was to analyze the actions that favor the use of discursive labels in the writing of texts by the students of the College Particular San Javier, Comuna San Javier, Maule-Chile Region. Methodologically, the quantitative approach was used, type field with descriptive design. As findings, the knowledge that teachers and students have about the use of discursive labels for writing texts was diagnosed; the most frequent functions that the discursive labels perform in the writing of written texts were identified, the discursive deficiencies produced by their scarce or inappropriate use were determined; finally, the actions that favor the incorporation of discursive labels in the writing of texts by the students under study were defined.

KEYWORDS: Labels. Discursive. Writing. Texts.

\section{Introducción}

La necesidad de aprender la información, de mantener el conocimiento a través del tiempo y el espacio parecen ser cosas naturales. Sin embargo, el individuo concibió la escritura, en lo extenso, en una cadena de períodos iniciadores y en un amplio proceso de acomodo de la imagen a la idea primero y, después a los sonidos. Por consiguiente, la escritura es el archivo de la memoria de la humanidad (SANTANDER, 2016). De esta manera, los textos escritos han ejercido una gran influencia sobre toda la población, especialmente, en el contexto educativo formal. Por lo tanto, la estructura de un escrito era estimada como un producto de simple en codificación o transcripción de la oralidad, actualmente se visualiza que el escenario de comunicación del discurso escrito es completamente diferente al oral, en virtud que, no están presentes, al mismo tiempo, el escritor y el lector.

Se entiende, por consiguiente, que el lenguaje no solo es utilizado para transmitir una información sino, también, para crear una versión de la realidad que acontece en un determinado entorno y, que tiene un marco de referencia en las diferentes formas de publicaciones y manifestaciones, donde se hace evidente el empleo de elementos lingüísticos invariables, como las llamadas etiquetas discursivas, las cuales cohesionan diferentes enunciados y guían las inferencias que se generan a partir de la interpretación del discurso. Para SALAZAR (2016), las etiquetas discursivas representan:

Un tipo de elementos fóricos que permiten hacer referencia a un fragmento anterior (anáfora) o posterior (catáfora) al tiempo que lo categorizan con un sustantivo 
(encapsulación). La importancia de este mecanismo no solo se encuentra en el análisis del discurso, sino también en el aprendizaje de una escritura coherente y cohesiva.

De este modo, se expresa que la eficacia y la coherencia de un texto resultan en parte, por la adecuada utilización de las etiquetas discursivas en la redacción de textos, en el caso que ocupa esta investigación, de los académicos. En otras palabras, la utilización lógica y pertinente del lenguaje escrito para generar un producto coherente y apropiado. Con base a lo anterior, SALAZAR (2016) expone que el etiquetaje discursivo se enmarca como "un procedimiento textual que incide en dimensiones tan diversas como la gestión del tópico discursivo, la organización retórica del texto, la conexión internacional o el poder persuasivo del discurso" (p.37). Por lo cual se entiende, que el interés de investigar en la conducta de los mismos en la escritura en situaciones académicas surge del rol clave en la disposición de las derivaciones y, en el proceso de la producción de textos. Al respecto, CALATRAVA (2016), que:

A nivel de las instituciones educativas se generan una gran variedad de escritos con formatos y características especialmente estereotipadas, siendo cotidianas las demandas como elaboración de informes, ensayos, investigaciones, entre otros y, quienes participan en ellas de uno u otro modo necesitan manipular eficientemente la diversidad textual para la producción de textos que tengan cohesión y coherencia. Sin embargo, lamentablemente, estas competencias no siempre están cabalmente desarrolladas (p. 55).

Lo referido por el autor anterior es sustentado por CÁRDENAS (2015), en un estudio realizado en la Universidad Santa María La Vieja, Panamá en el que indica "los estudiantes de educación media presentan dificultades para producir textos argumentativos, exhibiendo deficiencias para condensar el contenido de segmentos discursivos de manera coherente y cohesionado" (p. 23).

De igual manera, en Chile se han realizado estudios relacionados con la producción de textos, quedando en evidencia en el marco del Primer Estudio de Comportamiento Lector a Nivel Nacional (CNCA, 2015), citado por LEYTON; PARDO (2016), en el cual “el 33\% de los estudiantes presenta dificultades para generar de forma global un texto, reflexionar y evaluar los contenidos de lo leído, entre otras tareas de lectura y escritura" (p. 4). Significa que los estudiantes carecen de las competencias básicas de análisis, interpretación y percepción de la lectura y por consiguiente en la generación coherente y lógica de producciones escritas.

Así mismo JIMÉNEZ (2016), en su investigación titulada "Acciones para el mejoramiento en la redacción de los estudiantes chilenos: Una propuesta de revisión desde la 
lingüística del texto", tuvo como objetivo proponer acciones que favorezcan la redacción realizada con escritos de estudiantes del nivel Medio Científico Humanista (Primero a Cuarto Medio). Como conclusiones, primeramente, el autor identificó dificultades en las redacciones, resumiéndose en los siguientes aspectos: puntuación distraída e inexperiencia de su utilización primordial; abundancia de subordinación inducida por el descuido en la puntuación; pobreza de ideas: insuficiente variedad y desmedida repetición; variación del orden lógico y sintáctico de la estructura oracional; desorden entre el título y contenido; ausencia de conocimiento en el uso del párrafo, en su estructura externa e interna; duplicación de palabras, barbaridades, inexactitud de sinónimos. En consecuencia, se precisa la incidencia en las deficiencias en la redacción, se muestran carencias a partir de la representación discursiva en su dimensión textual y de microcomposición lo que imposibilita la presencia de calidad en la redacción del texto.

En tal sentido, el autor propone acciones orientadas al uso de etiquetas discursivas, hiperónimos y encapsuladores que permitan generar relaciones de cohesión referencial en la redacción de textos. Se entienden que estos elementos estriban del contexto no solo para la caracterización de su referente, sino también para ultimar el significado conceptual de su núcleo nominal y el espacio vacío o esquemático que existe en la estructura semántica de los nombres que desempeñan esta función discursiva.

Dentro de los planteamientos expuestos se entiende que se requiere un manejo de elementos lingüísticos básicos para una adecuada construcción de textos. Por lo que se hace mención, específica, del uso de etiquetas discusivas, las cuales desempeñan un papel importante, fundamentalmente al otorgar cohesión y coherencia gramatical a las ideas en el discurso escrito, considerando desde la función cognitivo-discursiva, por una parte, la encapsulación o condensación del contenido y, por otra la categorización o conceptualización del mismo para otorgarle entidad al discurso.

Con referencia a la categorización de las etiquetas discursivas en función de los principales tipos de discurso, se señala que estas acceden a concentrar el contenido de una o más estructuras predicativas conceptualizándola como una entidad de segundo o tercer orden, manteniendo una estrecha relación con los diferentes tipos de textos; por lo que las clasifica en dos grandes grupos: las funciones informativas y las meta discursivas, considerando para ello criterios lingüístico-discursivos (SUÁREZ, 2017). Entendiendo que imponen un conjunto de marcas lingüísticas que especifican, condicionan, dan sentido a la comprensión y elaboración textual, respondiendo la correcta progresión de la información, al imponer a una cadena de proposiciones de orden, con la finalidad de alcanzar un fin determinado. 
Por consiguiente, a partir de la comprensión de la utilidad de la enseñanza y aprendizaje de uno de los mecanismos de cohesión léxica, las etiquetas discursivas, como técnica de escritura académica, se plantea la realidad de los estudiantes pertenecientes al cuarto nivel Medio Científico Humanista del Colegio Particular "San José”, ubicado en la Comuna de San Javier de la Región del Maule-Chile, entendiendo que se requiere generar en estos una mayor atención en las producciones escritas, localizándola como objeto de aprendizaje en el contexto comunicativo, en virtud que están próximos a culminar la etapa de colegiatura para su ingreso en las instituciones de educación superior, en el cual van a demandar la construcción de unidades de significado coherentes de mayor complejidad, para avanzar en su proceso educativo.

Por ello, de manera general el objetivo de este trabajo de investigación persiguió establecer el funcionamiento, a partir de las acciones que favorezcan el uso de las etiquetas discursivas en la redacción de textos, por parte de este sector de estudiantes, discurrir como habilidad de escritura que admita representar al texto como unidad de comunicación lingüística organizada y relacionada entre sí y, que de manera explícita o implícita, contribuyan en su construcción desde las perspectivas sintáctica, pragmática y semántica en correspondencia con las propiedades de los textos.

\section{Metodología}

El tipo corresponde a una investigación de campo descriptiva en la que la recolección de datos surge directamente de los sujetos indagados o de la realidad en la cual suceden los hechos (datos primarios). De allí que el diseño tenga un carácter no experimental (ARIAS, 2016). Por otra parte, para sustentar la investigación se apoya en fuentes documentales, a lo cual BAVARESCO (2016), señala que “constituye prácticamente la investigación que da inicio a casi todas las demás, por cuanto permite un conocimiento previo o bien el soporte documental bibliográfico vinculante al tema de estudio [...] (p. 27). La contribución documental sustenta el desarrollo de cada una de las variables presentando un marco teórico que permite al investigador sustentar o refutar las consideraciones allí expresas.

Esta recopilación de información es necesaria para cumplir el propósito de revisar el problema y ofrecer alternativas viables, permitiendo indagar acerca del conocimiento que tienen los docentes y estudiantes sobre el uso de etiquetas discursivas para la redacción de textos, así como las distorsiones y deficiencias discursivas que produce la escasa o inapropiada incorporación de las mismas en el discurso escrito, para a partir de estos datos 
generar acciones que logren afianzar la construcción de textos en los cuales se permita realzar, no solo la coherencia y cohesión, sino la presencia de los diferentes elementos que caracterizan a una buena redacción.

Las preguntas orientadoras del proceso investigativo fueron: ¿Qué conocimiento tienen los docentes y estudiantes pertenecientes al cuarto nivel Medio Científico Humanista del Colegio Particular "San José”, ¿sobre el uso de etiquetas discursivas para la redacción de textos?; ¿Cuáles son las funciones frecuentes que desempeñan las etiquetas discursivas en la redacción de textos escritos?; ¿Cuáles deficiencias discursivas produce el uso escaso o inapropiado de las etiquetas discursivas en la redacción de textos escritos por parte de los estudiantes del cuarto nivel Medio Científico Humanista del Colegio Particular "San José"? Y; ¿Qué acciones favorecerán la incorporación de las etiquetas discursivas en la redacción de textos por parte de los estudiantes del cuarto nivel Medio Científico Humanista del Colegio Particular "San José"?

Con relación a los sujetos de intervención, se realizó, inicialmente, una reunión preparativa general, de esta manera, la población a la cual el investigador aplica las generalizaciones obtenidas para intentar formular afirmaciones que sean valederas para ese conglomerado; quedando conformada por seis (6) docentes y, veintidós (22) estudiantes del cuarto nivel Medio Científico Humanista que desarrollan la asignatura Lenguaje y Comunicación.

Se resalta que, a partir de la selección, las personas, después de ser informadas de las características del estudio y de solicitarle su colaboración, deben manifestar de manera voluntaria su disposición a participar en la investigación, esto se constata en el formulario denominado Consentimiento informado. Este representa la manifestación de la autonomía del personal que participa de manera voluntaria en el estudio, correspondiendo a la base legal y ética de la investigación.

Sobre las técnicas se utilizaron las siguientes: (a) Análisis documental, para examinar diferentes documentos relevantes y necesarios para así sustentar la teoría del problema en estudio; (b) Encuesta, dirigida a los docentes, en este sentido se hizo uso de su forma estructurada y, es definida por el HURTADO y TORO (2016), como "una técnica que pretende analizar, obtener información que suministra un grupo o muestra de sujetos a cerca de sí mismo, o en relación con un tema en particular" (p. 72).

Esta facilita la obtención de datos exactos confiables a través del contacto con la realidad, contribuyendo en la realización del diagnóstico que describió la situación objeto de estudio y; (c) Práctica Calificada, dirigida a los estudiantes, esta es definida por MARTÍNEZ 
(2015) como aquel que "permite comprobar las habilidades y destrezas adquiridas por el alumnado para desarrollar una determinada tarea, representativa de la conducta que desea medirse" (p. 34).

Se indicó para toda la muestra el mismo tema que debían desarrollar en el tiempo estipulado. Las veintidós (22) redacciones debían tener una página y media de extensión sobre algún problema de Chile y sus posibles soluciones, concibiéndose como un texto formal académico. En tal sentido, se entiende que las composiciones solicitadas cumplen el objetivo de informar sobre un problema y disuadir al lector sobre una posición en torno a este (causas, consecuencias, soluciones, entre otros), tal como lo hace un ensayo para cualquier curso educativo.

Se seleccionaron los siguientes instrumentos: Para atender la técnica de Análisis documental: (a) Fichas; (b) Computadoras y sus unidades de almacenajes y; (c) la Matriz de Análisis, a través de esta se extrae la información de los documentos revisados que fundamentan este estudio. Para los docentes: se diseñó un cuestionario estructurado con ítems preguntas cerradas con opción múltiple, en alguna de ellas se solicita ampliar la respuesta, con el propósito de poder facilitar el análisis de los datos, el cual permitió obtener la información correspondiente sobre el conocimiento de las etiquetas discursivas para la redacción de textos. Para los estudiantes: se considera la aplicación de la Valoración Descriptiva de Prácticas como establece TORRES (2015) bajo la incorporación de etiquetas discursivas, vinculándolas con los estándares de cohesión, coherencia e informatividad en la redacción de textos que poseen los estudiantes. El resultado de la lectura de las producciones escritas se registra en una Rubrica Analítica para este tipo de textos.

La Validez del Instrumento según lo establece HERNÁNDEZ, FERNÁNDEZ y BAPTISTA (2016), fue a través de contenido o juicios de expertos, con el propósito de obtener información y comprobar si los ítems reflejan claramente los objetivos que se pretenden lograr. En este sentido se seleccionaron dos especialistas en las áreas de metodología y español, a los que se les hizo llegar mediante comunicación escrita el instrumento a ser evaluado y la hoja de observación con sus respectivas instrucciones; se tomaron en cuenta la pertinencia, claridad y coherencia de cada uno de los ítems elaborados, posteriormente, a partir de ellos se realizaron las observaciones, las cuales se incorporan para luego ser aplicado a la muestra de estudio.

Al considerar la técnica de análisis de datos, se postula el criterio de MÉNDEZ (2015), para ello, posterior a la aplicación de los instrumentos, estos son revisados, se elabora una matriz de doble entrada para la transcripción de las respuestas dadas en cada ítem apoyado en 
el programa SPSS versión 11.5, determinando las frecuencias simples y porcentuales en cada alternativa de respuesta ofrecida. Seguidamente se procedió a la elaboración de cuadros descriptivos y, a realizar la interpretación y análisis de la información recabada, para la cual se utilizó el análisis descriptivo basado en la interpretación porcentual confrontando la relación con el marco teórico general estableciendo su relación con los objetivos y las variables, destacando los aspectos más relevantes encontrados.

\section{Resultados}

Con relación a los Docentes: El 67 \% de los entrevistados eran del sexo femenino y el $33 \%$ masculino; además el $50 \%$ se encontraba en el intervalo de edad 32-41 años y, el $50 \%$ entre 42-51 años. Estos expresaron que no tienen un pleno conocimiento sobre las posibilidades de funcionamiento cognitivo de las etiquetas discursivas (ED) como un mecanismo textual con capacidad de organización del texto, por lo que no la incorporan en la práctica educativa. No obstante, señalan que sí orientan el uso de otros mecanismos que permitan la cohesión y coherencia en los textos escritos.

Por otra parte, el $100 \%$ de los docentes afirman que la redacción en los estudiantes genera gran preocupación por el dominio inadecuado de las competencias de escritura, imposibilitándolo el logro de una efectiva comunicación escrita, dentro de la variedad académica, que permita la trasmisión fluida de sus ideas y la construcción final de conocimiento. Asimismo, un $87 \%$ de los docentes entrevistados sostiene que los estudiantes plantean el uso de la redacción con fines casi exclusivamente evaluativos, la dificultad para lograr la coherencia del texto escrito se relaciona con el hecho de que el discurso generado puede no regular la tarea al quedar superado por un fluir desordenado de ideas ante la exigencia percibida de la evaluación.

Por ello, dentro de las principales deficiencias discursivas que se indican que se presentan en la redacción de textos escritos por parte de los estudiantes están: $33 \%$ falta de claridad y orden, $33 \%$ incoherencia, $17 \%$ redundancia y $17 \%$ falta de consistencia. Estos datos hacen estimar que no todos los docentes en la dinámica educativa conducen un proceso que permitan al participante generar productos escritos, desde la incorporación de mecanismo textuales, desarrollando en el corpus del escrito enlaces léxico-cohesivos con relación semántica que resalte como mecanismo textual, la capacidad de organización del texto.

En cuanto a los resultados valoración descriptiva de prácticas aplicado a los estudiantes: el corpus estudiado correspondió a veintidós (22) redacciones tipo ensayo, el 
tema estuvo orientado a redactar algún problema de Chile y sus posibles soluciones, permitiendo disuadir al lector sobre una posición en torno a este (causas, consecuencias, soluciones, entre otros). Para ello, se tomó en cuenta tres de los estándares que se valoran a partir del producto escrito final, a saber: la cohesión, la coherencia y la informatividad; entendiendo que éstas proporcionan capacidad de organización del texto, siendo esto el elemento donde reside en realidad la relevancia de la etiqueta discursiva como recurso léxico y mecanismo textual.

Primera Fase: La producción de textos se valoró mediante niveles que se adaptaron para poder identificar cómo el estudiante empleaba las etiquetas discursivas (ED), a través de sus diferentes funciones en el contenido del texto, así:

Nivel 1. El texto no presenta ED que permita la cohesión y coherencia;

Nivel 2. El texto presenta algunas ED que permita la cohesión y coherencia, se repiten palabras de forma excesiva;

Nivel 3. El texto presenta adecuados ED que permita la cohesión y coherencia, pero repite más de dos veces algunas palabras;

Nivel 4. El texto presenta adecuados ED que permita la cohesión y coherencia.

Tabla 1 - Resultados individualizados presencia ED en el texto

\begin{tabular}{|c|c|c|}
\hline Estudiante & Nivel & Observaciones \\
\hline E1 & 1 & $\begin{array}{l}\text { Repetición excesiva de palabra "país". Falta de signos de puntuación entre } \\
\text { proposiciones. Ausencia de ED }\end{array}$ \\
\hline E2 & 1 & Ausencia de ED y de mecanismos de cohesión. Repite los términos "y", "también”" \\
\hline E3 & 2 & $\begin{array}{l}\text { Repetición moderada de palabras "lugar", “tiempo". Uso inadecuado signos de } \\
\text { puntuación. Presencia de algunas ED que permita la cohesión textual }\end{array}$ \\
\hline E4 & 3 & $\begin{array}{l}\text { Repetición modera de palabras "estas", "que". Uso adecuado se signos de } \\
\text { puntuación. Presenta adecuados ED que permita la cohesión y coherencia }\end{array}$ \\
\hline E5 & 1 & Ausencia de ED y mecanismos de cohesión. Repetición de excesiva "también" \\
\hline E6 & 2 & $\begin{array}{l}\text { Uso inadecuado de signos de puntuación. Empleo excesivo de los demostrativos } \\
\text { "estos". Presencia de algunas ED que permita la cohesión textual }\end{array}$ \\
\hline E7 & 3 & $\begin{array}{l}\text { Empleo ED de forma correcta, así como de otros mecanismos de cohesión como } \\
\text { pronombres relativos (que), posesivos ( } \mathrm{su} \text { ) y demostrativos (estos). }\end{array}$ \\
\hline E8 & 1 & $\begin{array}{l}\text { Repetición excesiva de las palabras "accidente", "autos", "tranque", } \\
\text { "contaminación". Ausencia de signos de puntuación. Ausencia de ED. }\end{array}$ \\
\hline E9 & 3 & $\begin{array}{l}\text { Empleo de mecanismos cohesivos de manera correcta principalmente pronombres } \\
\text { de tipos: relativos, posesivos, demostrativos. Signos de puntuación empleados } \\
\text { adecuadamente. Presenta adecuados ED que permita la cohesión y coherencia, pero } \\
\text { repite más de dos veces algunas palabras. }\end{array}$ \\
\hline E10 & 1 & $\begin{array}{l}\text { No posee ningún mecanismo de cohesión ni siquiera el básico que son los signos de } \\
\text { puntuación. Redactada en primera persona de plural y en segunda del singular. El } \\
\text { texto no presenta suficientes ED que permita la cohesión y coherencia. }\end{array}$ \\
\hline
\end{tabular}




\begin{tabular}{|c|c|l|}
\hline E11 & 1 & $\begin{array}{l}\text { Repetición de palabra "el celular" y "conector" también". El texto no presenta } \\
\text { suficientes ED que permita la cohesión y coherencia. }\end{array}$ \\
\hline E12 & 2 & $\begin{array}{l}\text { Repetición excesiva del pronombre relativo "que" y del demostrativo "este". El } \\
\text { texto } \\
\text { presenta algunas ED que permita la cohesión y coherencia. }\end{array}$ \\
\hline E13 & 1 & $\begin{array}{l}\text { Uso excesivo de la conjunción "y", texto no presenta suficientes ED que permita la } \\
\text { cohesión y coherencia. }\end{array}$ \\
\hline
\end{tabular}

Fuente: Elaboración propia

Tabla 2 - Resultados individualizados presencia ED en el texto (continuación)

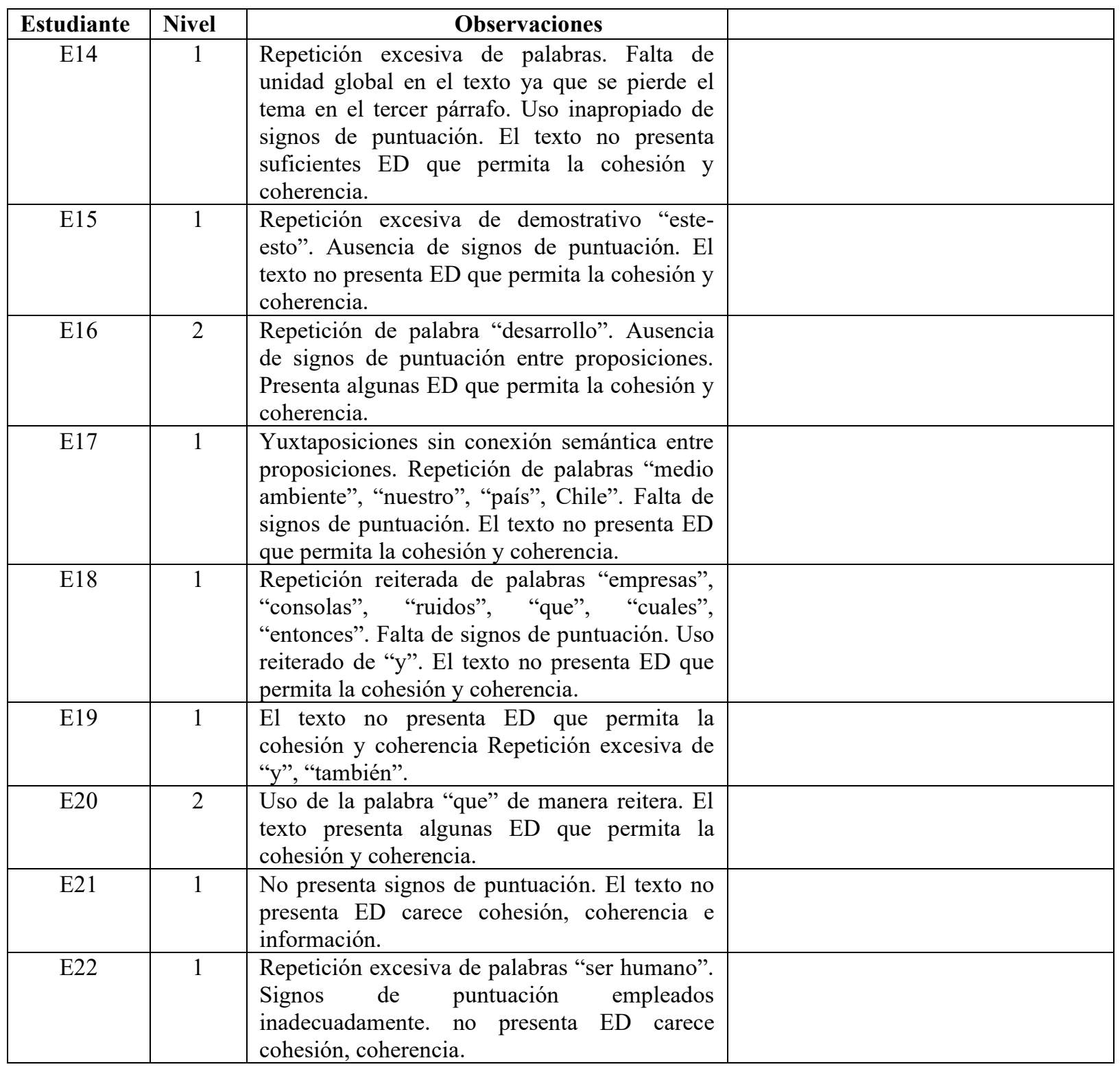

Fuente: Diagnóstico, elaboración propia 
Tabla 3 - Distribución de frecuencia presencia ED en el texto

\begin{tabular}{|c|c|c|}
\hline Nivel presencia ED & Cantidad de Estudiantes & Resultado (\%) \\
\hline Nivel 1 & 14 & 63 \\
\hline Nivel 2 & 5 & 23 \\
\hline Nivel 3 & 3 & 14 \\
\hline Nivel 4 & 0 & 0 \\
\hline Total & $\mathbf{2 2}$ & $\mathbf{1 0 0}$ \\
\hline
\end{tabular}

Fuente: Diagnóstico, elaboración propia

De acuerdo a los datos expuestos se interpreta que el $63 \%$ de los estudiantes se encuentran en el Nivel 1, Insuficiente, referido a la producción de textos que no presenta ED que permita la cohesión y coherencia, el $23 \%$ en el Nivel 2, Mínimo, en los cuales el texto presenta algunas ED que permita la cohesión y coherencia, repitiéndose palabras de forma excesiva y; el $14 \%$ en el Nivel 3, Satisfactorio, aquellos que generaron un texto con adecuada utilización de las ED confiriéndole cohesión y coherencia al mismo, sin embargo, persiste la repetición de algunas palabras. Segunda Fase: Se presenta algunas de las valoraciones cualitativas generadas en la revisión de los textos que permitieron la identificación de las deficiencias discursivas que produce el uso escaso o inapropiado de las etiquetas discursivas en la redacción de las producciones escritas:

Tabla 4 - Deficiencias por el uso escaso o inapropiado de las etiquetas discursivas en la redacción de las producciones escritas

\begin{tabular}{|l|l|}
\hline \multicolumn{1}{|c|}{ Deficiencias Discursiva textual } \\
\hline & Repetición léxica, Redundancia, incorrecta identificación de las entidades textuales \\
\hline & Referencias lejanas del texto. Falta de cohesión textual. No hay presencia de unidad en el párrafo \\
\hline $\begin{array}{l}\text { Limitada estructuración informativa (Empleo de palabras con la misma raíz en poco espacio) Uso de } \\
\text { categorías textuales que no corresponden a etiquetas discursivas }\end{array}$ \\
\hline & Desconocimiento de la ubicación textual de la etiqueta discursiva \\
\hline
\end{tabular}

Fuente: Elaboración propia

\section{Discusión}

No existe un pleno conocimiento sobre las posibilidades de funcionamiento cognitivo de las etiquetas discursivas como un mecanismo textual con capacidad de organización del texto. Este planteamiento sustenta lo indicado por CALATRAVA (2016), cuando señala que a nivel de las instituciones educativas se generan una gran variedad de escritos con formatos y 
características especialmente estereotipadas $\mathrm{y}$, requieren manipular eficientemente la diversidad textual. Estas competencias no están cabalmente desarrolladas, encontrándose la carencia en el uso de las etiquetas discursivas como elementos fóricos que permiten coherencia y cohesión en el discurso escrito.

Se percibe como la orientación y enseñanza para la redacción de textos está enfatizada en lecciones que se alinean desde una gramática normativa tradicional, sin conducir la reflexión y la pericia en torno a la función de los recursos lingüísticos que permitan una construcción textual de calidad, tal como lo indica CASTELLANO (2016). Por lo cual, es fundamental que operen intrínsecamente sus destrezas en los recursos gramaticales, ortográficos, conectores y etiquetas discursivas que afirmen y mantengan una gramática adecuada, que permita en la redacción de textos trabajar la coherencia y cohesión.

Los resultados evidencian la necesidad de generar acciones que le permitan al docente el uso de etiquetas discursivas en la enseñanza de la redacción de textos, teniendo como fin último el empoderar al estudiante hacía la apropiación de una red de conceptos básicos que fortalezcan su desempeño en el uso de las condiciones lingüísticas tanto morfológicas, sintácticas, semánticas, pragmáticas y cognitivas: a partir de la sufijación morfológica, hasta consideraciones de corte cognoscitivo, que el escritor principiante requiere llevar acabo en la planificación su texto, para enriquecer el contenido semántico de las entidades encapsuladas dándole fluidez a discurso escrito.

En función de los propósitos definidos para el estudio, se concluye, que el docente no posee conocimiento, por lo cual no hace uso de la enseñanza de las etiquetas discursivas para la creación de textos escritos como parte del desarrollo de la asignatura Lenguaje y Comunicación. Efectivamente, los educandos exhiben problemas para originar textos argumentativos, presentando dificultades en concentrar el contenido de segmentos discursivos de forma coherente y cohesionado. Esta estimación surge al valorar que en una misma redacción confluyen la falta de precisión léxica, coherencia textual, progresión temática, reglas gramaticales y ortográficas, entre otras, lo cual, evidentemente, provocan que el texto se perciba altamente deficiente.

Con base a todos los planteamientos los docentes consideran que, en virtud de reconocer la función y utilidad de las etiquetas discursivas, entendiendo que facilitan la redacción, concomitante al aumento del repertorio de expresiones e ideas que enriquecen el producto escrito dándole cohesión y, por consiguiente coherencia al escrito, es importante la incorporación de acciones que contribuyan con su uso, a través del ejercicio de este recurso para desarrollar procesos cognitivos desde la escritura. 
Por consiguiente, se corrobora la importancia de las funciones que desempeñan las etiquetas discursivas (ED) en la redacción de textos escritos como mecanismo anafórico de cohesión léxica, coadyuvando en la coherencia textual que limita la continuidad y unidad en la redacción, de acuerdo con (SALAZAR, 2016). A partir de ello se presentan las acciones permiten su uso apropiado: (1) contextualizar la unidad temática de la redacción, (2) atender la propiedad léxica y gramatical, cohesión textual, coherencia, claridad y, variedad léxica (3) Incorporación correcta de las ED, requiriendo para ello: la delimitación conceptual de ésta (uso de los principales patrones léxico-gramaticales), generación de procesos de nominalización (transformación de un bloque informativo en una entidad discursiva), desarrollar la integración conceptual (Integración del significado de un contenido conceptual complejo en una entidad conceptual menos compleja). Esto avalado por la postura de (SANTANDER, 2016).

\section{REFERENCIAS}

ARIAS, F. EI Proyecto de Investigación: Guía para su Elaboración. 7. ed. Caracas: Episteme, 2016. ISBN: 980-07-3868-1.

BAVARESCO, P. Procesos, estrategias y técnicas de aprendizaje. España: Síntesis. 2016.

CALATRAVA, P. La redacción en estudiantes universitarios. Un estudio de análisis. 2016. Tesis (Doctoral) - Universidad Central de Venezuela, Venezuela, 2016.

CÁRDENAS, L. Análisis en la redacción formal de textos por los estudiantes de educación media. 2015. Tesis (Doctoral) - Universidad Santa María La Vieja, Panamá, 2015.

CASTELLANO, O. Lingüística del texto. Madrid, España: Arcos y Libros: 2016.

HERNÁNDEZ, R.; FERNÁNDEZ, C.; BAPTISTA, P. Metodología de la investigación. México: McGraw-Hill, 2016

HURTADO, I.; TORO, J. Paradigmas y métodos de investigación en tiempos de cambio. Venezuela: CEC, S.A, 2016.

JIMÉNEZ, U. Acciones para el mejoramiento en la redacción de los estudiantes chilenos: Una propuesta de revisión desde la lingüística del texto. 2016. Tesis (Doctoral) - Universidad De Concepción, Chile, 2016.

LEYTON, A.; NÚÑEZ, J.; PARDO, D. ¿Cómo incide el programa de fomento lector en el desempeño académico de los estudiantes de los establecimientos educacionales intervenidos? Disponible: https://fundacionluksic.cl/wp-content/iploads/2016/11/InformeEstudio-Leyendo-en-Red-2016.pdf. Acceso: 10 jun. 2019. 
MARTÍNEZ, F. Redacción y ortografía. Una opción para todos. Heredia: Ediciones Marwal, 2015.

MÉNDEZ, U. Contra el método. Barcelona, España: Folio, 2015.

SALAZAR, M. Las estrategias de aprendizaje en el proceso de composición escrita. España: Valparaíso, 2016.

SANTANDER, P. Redacción y escritura. Caja de herramientas. Bogotá, Colombia: Xpress Estudio Gráfico Digital S.A, 2016.

SUÁREZ, L. Las etiquetas discursivas: categorización de entidades del discurso en la escritura profesional. 2017. Tesis (Doctoral) - Universidad de Córdoba, España, 2017.

TORRES, A. Construcciones conectivas que encapsulan para la escritura experta. 2015. Tesis (Doctoral) - Universidad de Córdoba, España, 2015.

\section{Cómo referenciar este artículo}

MARTÍNEZ, M.; MOTA, K.; QUIÑONEZ, J. Uso de etiquetas discursivas en la redacción de textos. Revista on line de Política e Gestão Educacional, Araraquara, v. 25, n. 1, p. 240253, jan./abr. 2021. e-ISSN:1519-9029. DOI: https://doi.org/10.22633/rpge.v25i1.14727

Enviado: $10 / 11 / 2020$

Revisiones necesarias: $20 / 11 / 2020$

Aprobado: 20/12/2020

Publicado: 02/01/2021 